\title{
Replica Selection in the Globus Data Grid
}

\author{
Sudharshan Vazhkudai, ${ }^{1}$ Steven Tuecke,${ }^{2}$ and Ian Foster ${ }^{2}$ \\ ${ }^{1}$ Department of Computer and Information Science \\ The University of Mississippi \\ chucha@john.cs.olemiss.edu \\ ${ }^{2}$ Mathematics and Computer Sciences Division \\ Argonne National Laboratory \\ \{tuecke,foster\}@mcs.anl.gov
}

\begin{abstract}
The Globus Data Grid architecture provides a scalable infrastructure for the management of storage resources and data that are distributed across Grid environments. These services are designed to support a variety of scientific applications, ranging from high-energy physics to computational genomics, that require access to large amounts of data (terabytes or even petabytes) with varied quality of service requirements. By layering on a set of core services, such as data transport, security, and replica cataloging, one can construct various higher-level services. In this paper, we discuss the design and implementation of a high-level replica selection service that uses information regarding replica location and user preferences to guide selection from among storage replica alternatives. We first present a basic replica selection service design, then show how dynamic information collected using Globus information service capabilities concerning storage system properties can help improve and optimize the selection process. We demonstrate the use of Condor's ClassAds resource description and matchmaking mechanism as an efficient tool for representing and matching storage resource capabilities and policies against application requirements.
\end{abstract}

Keywords: Data Grid, Grid Computing, Replica Selection, Globus

\section{Introduction}

An increasing number of scientific applications ranging from high-energy physics to computational genomics re- quire access to large amounts of data-currently terabytes and soon petabytes - with varied quality of service requirements. This diverse demand has contributed to the proliferation of storage system capabilities, thus making storage devices an integral part of the Grid environment and thereby constituting the Data Grid. The Globus [6] Data Grid architecture is an effort to standardize access to the multitude of storage systems spread across the Grid environment. It attempts to abstract these diverse elements of the data grid by providing a set of core services that can be used to construct a variety of higher-level services [2].

In this paper, we first describe the basic Globus Data Grid architecture, explaining briefly the various components. We then describe the process of identifying characteristics of interest about a storage replica resource, and we illustrate the mechanism with which these characteristics can be published. Next, we explain the architecture of replica selection, a higher-level service, built by using core services provided by the Globus data grid. We conclude by presenting the use of Condor's classified advertisements (ClassAds) and matchmaking mechanisms [ 9 ] as an elegant matching and ranking tool in a storage context.

\section{Architecture of Data Grid}

The Globus data grid is organized into two layers, namely, core services and higher-level services that are built using the core services. The working hypothesis is that this hierarchical organization will make it possible to reuse services and code across a variety of applications and tools, so that, for example, both application-specific replica management solutions and sophisticated storage management systems such as the Storage Request Broker (SRB) [3] can share common low-level mechanisms. 


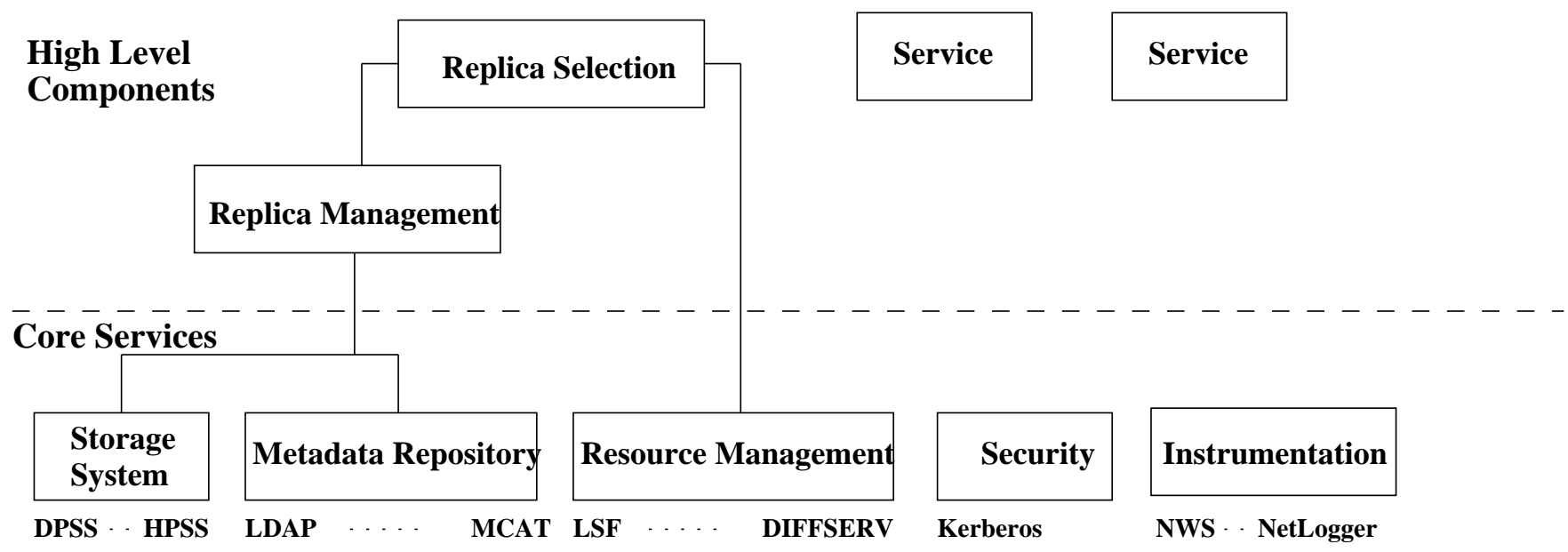

Data Grid Specific Services

Generic Grid Services

Figure 1. Data Grid Architecture [2].

\subsection{Core Services}

Core Data Grid services (Figure 1) seek to abstract the multitude of storage systems that exist in Grid environments, so that higher-level services — and applications - can access those storage systems uniformly. These core services include the following:

- Storage Systems and Data Access - Storage system and data access services provide basic mechanisms for accessing and managing the data located in storage systems. These mechanisms provide abstractions for uniformly creating, deleting, accessing and modifying file instances across storage systems, regardless of their physical location. They target secondary and archival storage systems such as Unix file systems, High Performance Storage System (HPSS) [П], and Unitree and can also be used to access more sophisticated systems such as SRB.

- Metadata Access - The metadata access service provides mechanisms to access and manage information about the data stored in storage systems. Various kinds of metadata are provided by this service:

1. Application Metadata describes the contents of the file, the circumstances under which the data was collected, and various details used by the application.

2. Replica Metadata describes the mapping between file instances and particular replica locations.
3. System Configuration Metadata describes the capabilities of storage systems, thus providing information on the fabric of the Data Grid.

The metadata service further provides a uniform method with which the various metadata can be published and accessed [2].

These services themselves build on basic security and information services provided by the Globus Toolkit [6].

\subsection{Higher-Level Services}

The core Data Grid services can be used to construct a variety of higher-level services (Figure 1). For example:

- Replica Management - Replica management is the process of creating or deleting replicas at a storage site. Most often, these replicas are exact copies of the original files, created only to harness certain performance benefits. A replica manager typically maintains a replica catalog containing replica site addresses and the file instances.

- Replica Selection - Replica selection is the process of choosing a replica from among those spread across the Grid, based on some characteristics specified by the application. One common selection criteria would be



\section{Storage System Functionality and Replica- tion}

Intelligent replica selection requires information about the capabilities and performance characteristics of a storage 
system [2]. We address this information discovery problem by leveraging machinery provided by the Globus Metacomputing Directory Service (MDS) [5], an information collection, publication, and access service for Grid resources.

The Globus MDS uses the Lightweight Directory Access Protocol (LDAP) [7] as its access protocol and LDAP object classes as its data representation, but it adopts innovative approaches to the problems of resource registration and discovery. Information about an individual resource or set of resources is collected and maintained by a Grid Resource Information Service (GRIS) daemon, which responds to LDAP requests with dynamically generated information and can be configured to register with one or more Grid Index Information Services (GIISs). Users will typically direct broad queries to GIIS to discover resources and then drill down with direct queries to GRIS to get up-todate, detailed information about individual resources.

Information in LDAP is organized in a tree structure referred to as a Directory Information Tree (DIT), with nodes in a DIT tree corresponding to the LDAP structured data types called object classes. Figure 3 depicts the DIT hierarchy of object classes that we have defined to describe storage systems in a Data Grid environment. We describe these object classes in the following section.

\subsection{Storage GRIS}

Each storage resource in the Globus Data Grid incorporates a Grid Resource Information Server, configured to collect and publish system configuration metadata describing that storage system. This information typically includes attributes such as storage capacity and seek times, and descriptions of site-specific policies governing storage system usage. Figure 2 shows the object class definition that we have developed for this data. Attributes are labeled as either "MUST CONTAIN" or "MAY CONTAIN," indicating whether or not they are mandatory.

This object class contains both dynamic and static attributes: attributes such as totalspace, availablespace, and mountPoint are dynamic, varying with various frequencies; attributes such as diskTransferRate, drdTime, dwrtime, and requirements are more static.

The requirements attribute is particularly interesting: it allows an administrator to specify the conditions under which the specified storage replica can be used, based, for example, on device utilization. For example, the requirements attribute could be a Boolean expression of maximum allowable storage and transfer bandwidth and can be specified using the ClassAd [9] mechanism. We discuss ClassAds in detail in a later section.

The data shown in Figure 1 is gathered in a variety of ways. We base our general approach on a generic

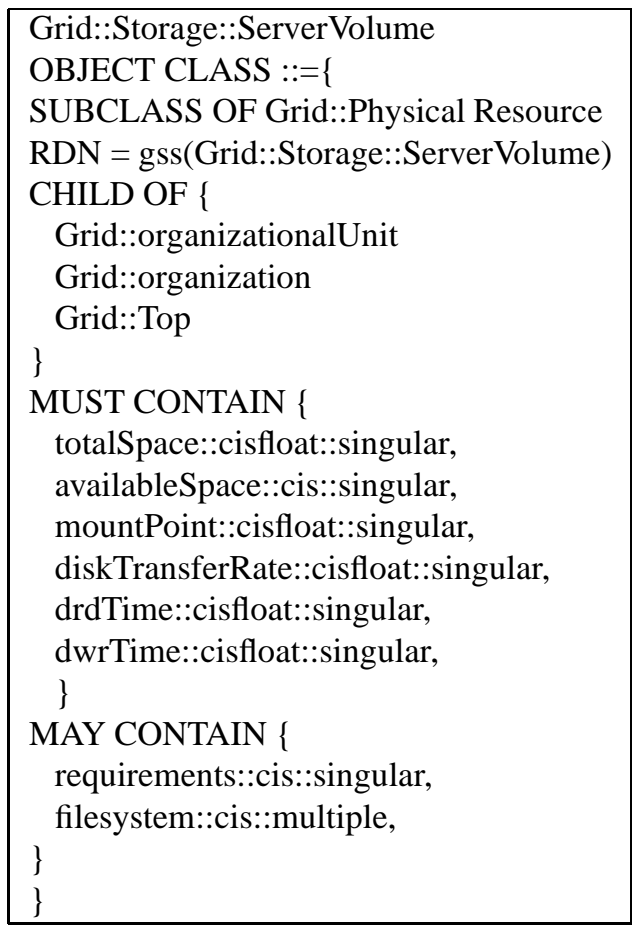

\section{Figure 2. System Configuration Metadata specification using LDAP object classes.}

LDAP-based GRIS server developed within the Globus project. The OpenLDAP server has a feature by which shell scripts ("shell-backends") can be executed at the backend in response to search queries; we use such scripts to gather dynamic attributes such as availablespace, mountPoint, and totalspace. Static attributes such as site usage policies and seek times can be specified by the administrator in a configuration file. Data collected in this manner is published in a suitable format (for example, LDIF 河).

\subsection{Data Access Service}

We are also interested in the speed of a storage system, or, rather, in the time that the storage system can be expected to take to deliver a replica. One approach to determining this information is to construct a performance model of the relevant components (e.g., see [10]). We favor an alternative approach in which historical information concerning data transfer rates is used as a predictor of future transfer times. In brief, storage systems are configured to provide information on their own behavior and performance. Attributes such as maximum achievable read and write transfer bandwidths across networks can help an application choose one replica over another. Such data can be obtained by the storage replica by monitoring their own 


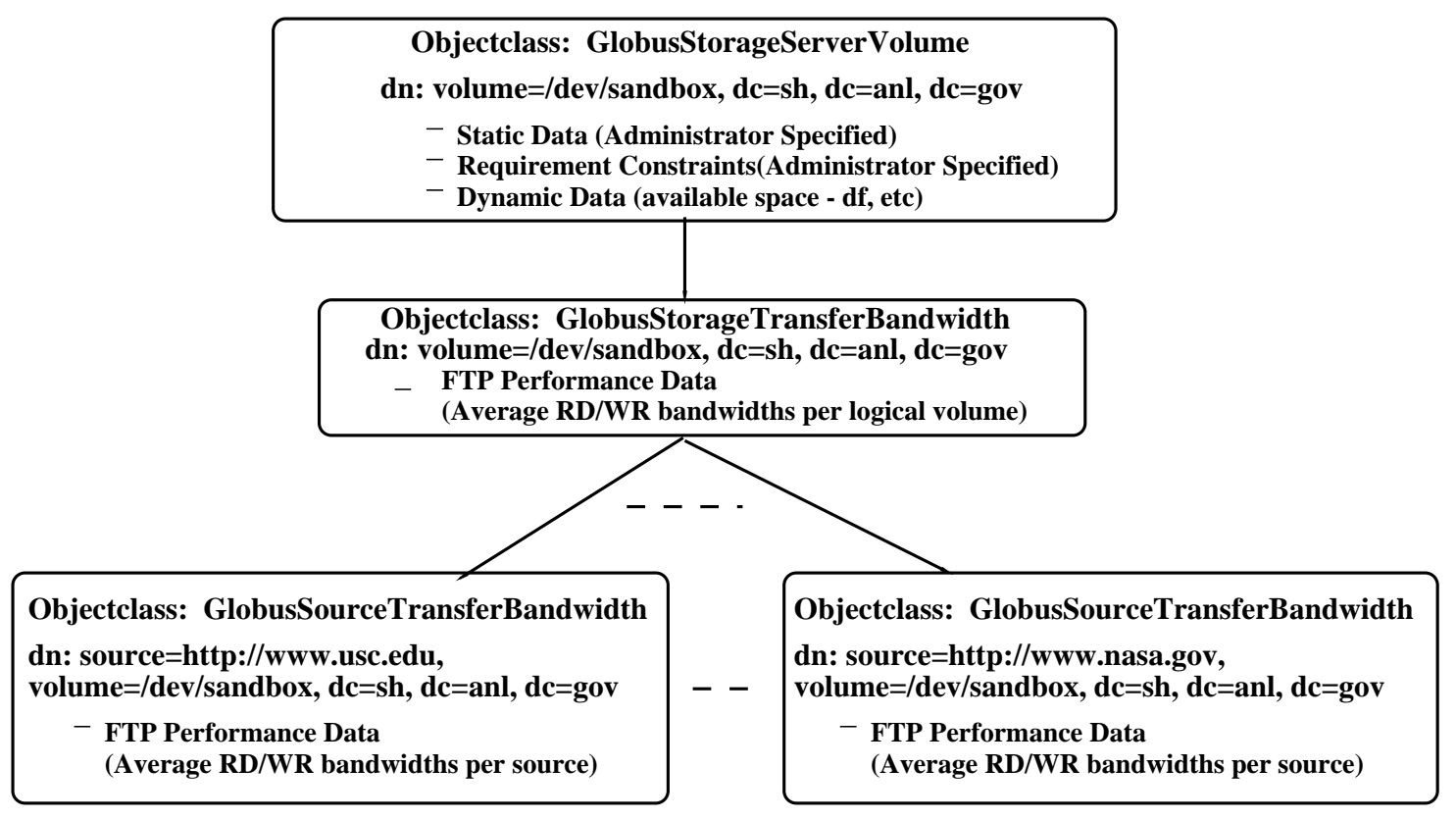

Figure 3. The directory information tree structure used in our storage system GRIS

performance. This feature can be extended further, to obtain statistical information based on the performance data, such as average transfer bandwidths and their standard deviations, that can help predict the behavior of a particular replica. Further, we expect that there will be significant reuse of storage servers by clients, thereby justifying performance information on a per source basis, which provides a client useful information on end-to-end transfer performance between the server and the client. For example, a simple heuristic of combining past observed performance with current load of server might give a client a reasonably good choice of server.

Figures 4 and 5, along with Figure 3, depict the object classes used to record performance data associated with a replica location. The object class in Figure $\Theta$ specifies a summary of transfer bandwidth performance for all replica transfers, and the object class in Figure 5 specifies the performance details from the replica location to particular source sites. We gather this performance data by using instrumentation incorporated in the GridFTP server [6][1].

\section{Classified Advertisements}

Classified Advertisements (ClassAds) [9] are an elegant matching mechanism used in the Condor high-throughput computing system to map resource capabilities against job requirements. ClassAds allow resources and jobs to advertise their capabilities and requirements as attribute-value pairs that can be matched. Two ClassAds match if the logical expressions contained in the "requirements" attribute in both of them is satisfied. This requirements attribute itself can be represented in terms of other attributes. Further, ClassAds provide a mechanism for ranking the matches based on some attribute value, or value computed from multiple attributes. Up until now, ClassAds have been used extensively in Condor for job placements. In this section, we present the use of ClassAds in a storage context.

The various storage attributes described in the preceding section can be represented as attribute-value pairs in a ClassAd. For example, the following is a simple ClassAd describing the capabilities of a storage resource:

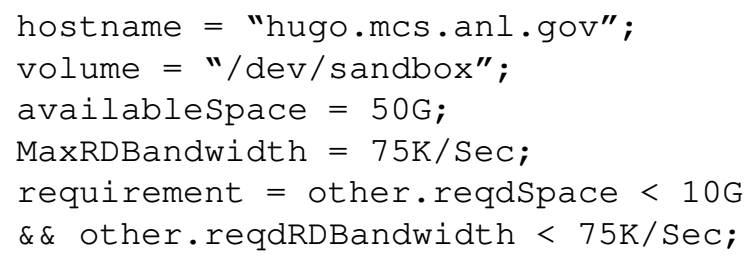

This ClassAd describes a volume of a storage resource by specifying its attributes. The ClassAd also specifies a usage policy enforced by the resource, whereby only applications requiring storage less than $10 \mathrm{~GB}$ and transfer bandwidths less than $75 \mathrm{~KB} / \mathrm{sec}$ are granted access. When two ClassAds are being matched, a MatchClassAd is created that contains both ClassAds. Each ClassAd can refer to the other ClassAd by using the "other" keyword.

A ClassAds representation of storage capabilities provides an efficient environment for matching, querying, and ranking requests. We will discuss the specifics of a request 




Figure 4. Performance data specification describing entire site characteristics, using LDAP object classes.

ClassAd and the matching process once we describe the replica selection mechanism.

\section{Replica Selection}

As noted above, a Data Grid provides a convenient environment for a community of researchers, interested in particular data sets, to maintain replicas of the data sets at their respective sites. Such an environment would provide both faster access and better performance characteristics. Replica selection is a high-level service provided by the Data Grid based on the core services. The replica selection process allows an application to choose a replica, from among those in a replica catalog, based on its performance and data access features.

An application that requires access to replicated data begins by querying an application specific metadata repository, specifying the characteristics of the desired data. The metadata repository maintains associations between representative characteristics and logical files, thus enabling the application to identify logical files based on application requirements. Once the logical file has been identified, the application uses the replica catalog to locate all replica locations containing physical file instances of this logical file, from which it can choose a suitable instance for retrieval.

The entity that identifies the suitable instance of a replicated file based on application requirements is referred to as

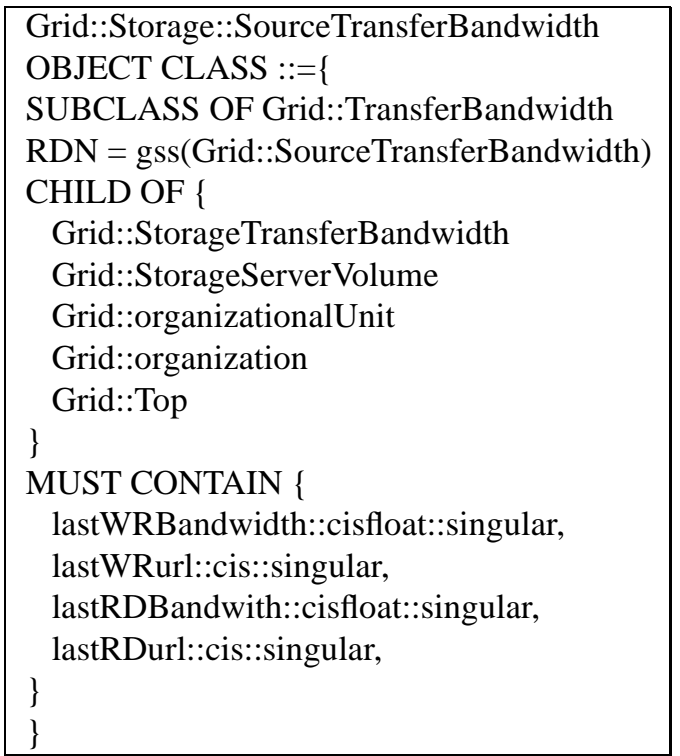

Figure 5. Performance data specification describing per source characteristics using LDAP object classes.

a broker. In effect, the responsibility of the broker is to map application requirements against storage resource capabilities. In the following section, we discuss the storage broker in detail.

\subsection{The Storage Broker}

In this section, we first delve into the details of the storage broker architecture. We then discuss the overall architecture, a decentralized selection process, and a few issues involved in its realization.

\subsubsection{Decentralized Selection Process}

Traditionally, resource brokers have adopted a centralized approach to resource management, wherein a single node is responsible for decision making. An example of such an environment is the Condor [8] high-throughput computing platform, wherein a central manager is responsible for matching resources against jobs. Obvious disadvantages to this approach are scalability and a single point of failure. Of course, Condor has an efficient recovery mechanism to address failure and has been proven to scale to thousands of resources and users.

But there is a more fundamental problem with this centralized approach when applied to Grids. In these highly distributed environments, there are numerous user communities and shared resources, each with distinct security requirements. No single resource broker is likely to be trusted 
by all of these communities and resources with the necessary information to make decisions. At the extreme, each user may need his or her own resource broker, because only that user has the authorization to gather all of the information necessary to make brokering decisions.

For this reason, we have designed a decentralized storage brokering strategy wherein every client that requires access to a replica performs the selection process rather than a central manager performing matches against clients and replicas.

\subsubsection{Architecture}

Figure 6 presents a snapshot of the Grid environment where storage resources are scattered and each client requiring access to a replica initiates a decentralized replica selection mechanism. As can be seen, there is no central point of control, and decision making is delegated to each and every client.

An application requiring access to a file presents its requirements as a ClassAd to the broker. These requirements might be as simple as a Boolean expression indicating the storage and transfer bandwidth required or may indicate more complex constraints on storage system type, state, and policy. The broker then performs the following sequence of actions:

\section{- Search Phase}

1. The broker attempts to find a suitable replica matching the application's requirements. To achieve this, it queries the replica catalog, which contains addresses of all replicas for each logical file.

2. The next step is to query each replica location. As we have seen before, this involves using LDAP searches to query GRIS servers associated with storage systems.

3. In response to the LDAP search, each storage system returns its capabilities and usage policies in the LDAP Information Format.

4. The broker collects the capabilities of all replica resources and proceeds to the matching phase.

\section{- Match Phase}

1. The replica capability data is converted into ClassAd format in preparation for invoking the Condor matchmaking mechanisms. We thus obtain a list of classified advertisements, representing the various replica sites.

2. The broker then performs a match of the application's requirement ClassAd against the list of replica capability ClassAds, obtaining a set of replica locations that satisfy the criterion.

3. The ClassAd ranking feature can be used to prioritize successful matches based on some attribute, specified by the application.

\section{- Access Phase}

1. Once a suitable replica has been identified, the file is accessed using a high-speed file transfer protocol, for example the GridFTP tools provided within the Globus Toolkit.

\subsection{An Example Application Request}

In Section 7 , we described a storage ClassAd that indicates its willingness to accept application requests that require space less than $10 \mathrm{~GB}$ and require a transfer bandwidth less than $75 \mathrm{~KB} / \mathrm{sec}$. In this section, we look at a user request ClassAd and how it is matched against the storage ClassAd.

An application might advertise its request to the broker as follows:

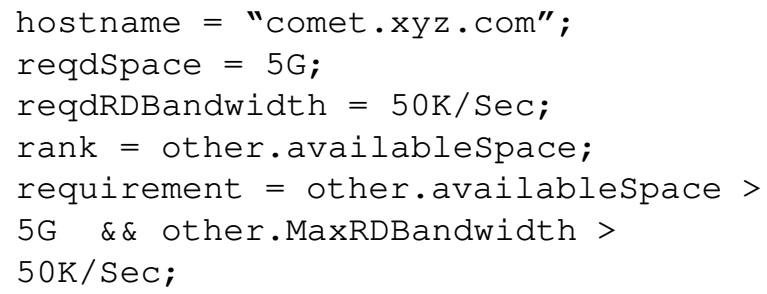

The application indicates its preference for a storage resource that has more than $5 \mathrm{~GB}$ available space and a maximum transfer bandwidth greater that $50 \mathrm{~KB} / \mathrm{sec}$. The broker, upon receiving such a request, attempts to contact all replica locations identified by the replica catalog, using LDAP search queries to request the attributes of interest: in this case, availablespace and MaxRDBandwidth. The broker thus uses the application ClassAd to build specialized LDAP search queries.

The results obtained via these LDAP queries are converted from LDAP Interchange Format (LDIF) into ClassAds and standard Condor mechanisms are invoked to match the application ClassAd with each ClassAd in the list of storage system ClassAds. (Note that the storage system ClassAds comprise their site specific usage policy, as mentioned in the sample storage ClassAd in Section 4 .) Any matched ClassAd can be further ranked by querying their rank attribute. In our case, we rank the replica servers based on their available space, thus obtaining the "best" match [7]. 




Figure 6. Storage Broker Architecture .

\section{Results}

We summarize our initial results based on our experience in building this prototype.

We have succeeded in building a replica selection broker and have demonstrated that this high-level Data Grid service can be built using the services provided by the Globus Data Grid Toolkit, such as: Storage GRIS, GridFTP and Replica Catalog.

We have further demonstrated the process of: Identifying characteristics of interest on a storage resource, based on Storage GRIS and GridFTP protocols; Classifying them as belonging to appropriate object classes according to LDAP; Gathering these attributes, via such mechanisms as shell backend scripts, tuning FTP servers and configuration files; Publishing the features in a suitable, efficiently queriable format using LDAP protocol.

We have explored the use of Condor ClassAds as a mechanism for expressing storage resource capabilities in the Grid environment. Although LDAP provides an equivalent method of publishing characteristics in attribute-value pairs, ClassAds provides a richer matching and ranking environment. Further, in our prototype implementation, we have demonstrated that the process of converting data, represented in LDAP format, into ClassAds is not cumbersome and is worth the effort. We have, in fact, developed primitive libraries to achieve the conversion of this attribute set.

\section{Conclusions and Future Directions}

Our current prototype implementation is primarily a proof of concept of the Globus Data Grid services. As the next logical step in its development, we need to demonstrate its applicability in a real application, thereby corroborating its usefulness. Further improvements can be made with regards to the information published in the storage GRIS. Finally, the statistical information published by the storage resource can be fed to an information service, such as the Network Weather Service [11], to perform predictive analysis of the behavior of storage resources.

\section{Acknowledgments}

This work was supported in part by the Mathematical, Information, and Computational Sciences Division subprogram of the Office of Advanced Scientific Computing Research, U.S. Department of Energy, under Contract W-31109-Eng-38; by the National Science Foundation; and by the NASA Information Power Grid program.

\section{References}

[1] B. Allcock, J. Bester, A.L. Chervenak, I. Foster, C. Kesselman, V. Nefedova, D. Quesnel, and S. Tuecke. Secure, Efficient Data Transport and 
Replica Management for High-Performance DataIntensive Computing. In In Mass Storage Conference, 2001.

[2] W. Allcock, A. Chervenak, I. Foster, C. Kesselman, C.Salisbury, and S. Tuecke. The Data Grid: Towards an Architecture for the Distributed Management and Analysis of Large Scientific Datasets. Journal of Network and Computer Applications, 2001. (to appear).

[3] C. Baru, R. Moore, A.Rajasekar, and M. Wan. The SDSC Storage Resource Broker. In Proceedings of CASCON'98, 1998.

[4] R.A. Coyne and R.W. Watson. The Parallel I/O Architecture of the High-Performance Storage System (HPSS). In IEEE MSS Symposium. IEEE Computer Society Press, 1995.

[5] S. Fitzgerald, I. Foster, C. Kesselman, G. von Laszewski, W. Smith, and S. Tuecke. A Directory Service for Configuring High-performance Distributed Computations. In Proc. 7th IEEE Symp. on High Performance Distributed Computing, pages 365-375. IEEE Computer Society Press, 1997.

[6] I. Foster and C. Kesselman. The Globus Project: A Status Report. In Proceedings of the Heterogeneous Computing Workshop, pages 4-18. IEEE Press, 1998.

[7] T.A. Howes and M.C. Smith. LDAP Programming Directory-Enabled Application with Lightweight Directory Access Protocol. Technology Series. MacMillan, 1997.

[8] M. Litzkow, M. Livny, and M. Mutka. Condor - A Hunter of Idle Workstations. In Proc. 8th Intl Conf. on Distributed Computing Systems, pages 104-111, 1988.

[9] R. Raman, M. Livny, and M. Solomon. Matchmaking: Distributed Resource Management for High Throughput Computing. In Proc. 7th IEEE Symp. on High Performance Distributed Computing. IEEE Computer Society Press, 1998.

[10] X. Shen and A. Choudhary. A Multi-Storage Resource Architecture and I/O, Performance Prediction for Scientific Computing. In Proc. 9th IEEE Symp. on High Performance Distributed Computing, pages 2130. IEEE Computer Society Press, 2000.

[11] R. Wolski. Dynamically Forecasting Network Performance Using the Network Weather Service. Journal of Cluster Computing, 1998. 